\title{
Double Mid-Latitude Dynamical Reconnection at the Magne- topause: an Efficient Mechanism Allowing Solar Wind to Enter the Earth's Magnetosphere
}

\author{
M. Faganello ${ }^{1}$, F. Califano ${ }^{2}$, F. Pegoraro ${ }^{2}$, T. Andreussi ${ }^{3}$ \\ 1 IIFS/PIIM, UMR 7345 CNRS Aix-Marseille University, Marseille, France ${ }^{2}$ Phys. Dept., University of Pisa, Pisa, \\ Italy $^{3}$ Alta SpA, Pisa, Italy
}

\author{
PACS 94.30.cp-First pacs description \\ PACS 52.35. Mw - Second pacs description \\ PACS 94.30.-d - Third pacs description
}

\begin{abstract}
Three-dimensional simulations of the Kelvin-Helmholtz (KH) instability in a magnetic configuration reproducing typical conditions at the flank Earth's magnetosphere during northward periods show the system's ability to generate favorable conditions for magnetic reconnection to occur at mid-latitude. Once these conditions are established, magnetic reconnection proceeds spontaneously in both hemispheres generating field lines that close on Earth but are connected to the solar wind at low-latitude, allowing direct entrance of solar wind plasma into the magnetosphere. These results are consistent with recent observations of $\mathrm{KH}$ vortices showing the signature of reconnection events occurring well outside the equatorial plane (Bavassano et al., 2010 Ann. Geophys. 28, 893 (2010)).
\end{abstract}

In the interaction between the solar wind and the Earth's magnetosphere different phenomena can cooperate at the magnetopause boundary where strong gradients are present and can provide free energy for the excitation of waves and instabilities. In such a complex magnetized system the interplay between different instabilities can have a "non-local" character due to the fact that the magnetic field lines, being frozen in the plasma over most of the configuration, can act as "rods" and transfer momentum and energy very effectively to locations far from the region where primary instabilities develop [1]. Eventually external constraints, such as the field lines being tied to the Earth, require that energy be released through the development of field line reconnection. In particular hydromagnetic large-scale instabilities such as the KelvinHelmholtz $(\mathrm{KH})$ instability in the equatorial region [2] can act as a forcing "at a distance" compared to the location where localized reconnection finally occurs. In this case including 3D effects and line tying provides a new perspective that tends to be obscured within $2 \mathrm{D}$ models and that can explain the transport properties of the magnetopause boundary during northward periods. In fact it has been shown that a mostly northward solar wind magnetic field is associated with a cold and dense plasma pop- ulation observed inside the Earth's magnetosphere plasma sheet, indicating the entrance of solar wind plasma into the magnetosphere [3]. Magnetic reconnection apperas to be a good candidate for explaining this efficient transport. In fact, due to the quasi-collisionless nature of space plasmas collisional cross field diffusion is ineffective, while collisionless diffusive processes associated to the observed wave-activity are believed to be too weak to explain the efficient mixing between the magnetosphere and the solar wind $[4-6]$. On the other hand reconnection, as the only mechanism able to change the global magnetic field topology, can allow different types of previously "unconnected" plasmas to mix $[7,8]$, thus explaining the entrance of solar wind plasma into the magnetosphere.

During northward magnetic field periods reconnection can occur in the lobe regions, between solar wind field lines draping over the sunward boundary of the magnetosphere and magnetospheric lines going from the Earth's poles to the tail [9]. In the case of reconnection proceeding almost simultaneously in both lobes, this double reconnection process could create magnetospheric-like flux tubes partially populated by solar wind plasma and has been proposed as a mechanism for the formation of the observed mixing layer $[10,11]$. Favorable conditions for 
reconnection during northward periods can also be created by the dynamics driven by large-scale $\mathrm{KH}$ vortices growing along the flank magnetopause near the equatorial plane [2], without being significantly inhibited by magnetic tension. Indeed, KH vortices spontaneously build up magnetic inversion layers where reconnection can occur [12-15] by stretching and advecting the magnetic field lines. These numerical results, obtained in a simplified $2 \mathrm{D}$ geometry, show that magnetic reconnection is able to connect the solar wind and magnetospheric regions on a time scale that is comparable to the $\mathrm{KH}$ scale provided the plasma model used for describing the evolution of the whole system includes at least two-fluid effects allowing reconnection to proceed on the correct time scale [16-18].

However it is known that in a $3 \mathrm{D}$ geometry the magnetic configuration stabilizes the KH instability at highlatitude. The resulting differential advection of the magnetic field lines suggests that favorable conditions for magnetic reconnection should be created at mid-latitude instead of at the equatorial plane $[19,20]$. Preliminar numerical studies have shown that magnetic reconnection can actually occur at mid-latitude and change the global topology [21]. Furthermore, observations of KH vortices developing in the equatorial plane showed the presence of particle distributions that were conjectured to be produced by magnetic reconnection occurring away from the equatorial plane [22] and not associated with reconnection between or inside vortices as expected in a $2 \mathrm{D}$ modeling of $\mathrm{KH}$ vortices.

In this letter we show that in a mostly northward magnetic configuration the differential advection of magnetic field lines, driven by the $\mathrm{KH}$ vortices that develop mainly in the equatorial region, creates current sheets at midlatitudes where reconnection eventually occurs. In general this spontaneous onset of reconnection proceeds in both hemispheres on the same time scale, thus creating magnetic flux tubes that are topologically connected to Earth at both poles but are embedded in the solar wind when crossing the low-latitude region. We may thus conclude that these magnetosphere-like flux tubes are partially populated by solar wind plasma and show that the quantity of plasma that is exchanged between flux tubes connected to the Earth and flux tubes connected to the solar wind is sufficient to explain the observed transport. In addition we show that this kind of double-reconnection process is not only boosted by the approximately northsouth symmetry, as in the case of the so-called double lobe reconnection $[10,11]$, but is actually related to the $3 \mathrm{D}$ dynamics of the system and to the consequent braiding of the magnetic field lines.

We adopt a 3D slab geometry, with the open inhomogeneity direction perpendicular to the magnetopause along the $x$ axis, the northward direction along the $z$ axis and the $y$-direction aligned along the solar wind direction. Periodic boundary conditions are used in the $(y, z)$ plane. We consider an ideal magnetohydrodynamic equilibrium configuration, varying in the $(x, z)$ plane and constant along

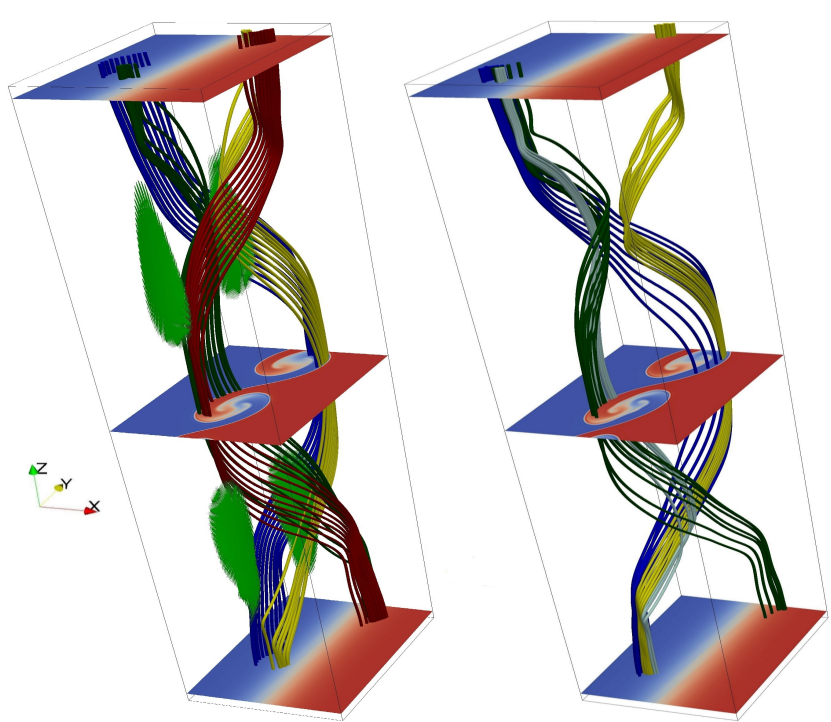

Fig. 1: (color online). Left frame: blue/red passive tracer in $x, y$-planes at $z=-180,0,+180$, light green current sheet isosurfaces defined by $|\mathbf{J}|>1.6$, and field lines showing the structures at $t=410$ of the X-line at $x \simeq 0, y \simeq$ $15,75 \lesssim z \lesssim 115$. Left frame: blue/red passive tracer in $x, y$ planes at $z=-180,0,+180$ and blue, green and ochre, and grey lines showing respectively pristine magnetospheric, oncereconnected and double-reconnected lines at $t=430$.

$y$, able to model the high-latitude stabilization of the $\mathrm{KH}$ instability. Stabilization is achieved by varying the characteristic scale length of the velocity gradient. We assume a nearly northward magnetic field lying in the $x, z$ plane and defined by the $y$-component of the vector potential,

$$
A_{e q, y}(x, z)=\frac{1}{2}\left[\frac{4}{3} x+\frac{L_{z}}{3 \pi} \sinh \frac{2 \pi x}{L_{z}} \cos \frac{2 \pi z}{L_{z}}\right]
$$

where physical quantities are normalized to the ion skin depth $d_{i}$, the ion cyclotron frequency $\Omega_{c i}$ and the ion mass $m_{i}$. In the simulations presented below we have taken the box dimensions as $L_{x}=60, L_{y}=30 \pi$ and $L_{z}=120 \pi$. The initial large-scale, sheared velocity field is thus a function of $A_{e q, y}(x, z)$ [23], $\mathbf{U}_{e q}(x, z)=$ $\left(U_{0} / 2\right) \tanh \left[A_{e q, y}(x, z) / L_{u}\right] \hat{\mathbf{y}}$, where $L_{u}=3$ is the halfwidth of the velocity shear layer. In order to focus on reconnection, we take constant and homogeneous density and temperature profiles so as to inhibit the development of other secondary instabilities [24-26]. The reference values of the sound and Alfvén Mach numbers are set equal to $M_{s}=U_{0} / C_{s}=1.3, M_{A}=U_{0} / U_{A}=1.0$, with $U_{0}=1.0$ and $U_{A}$ the value of the equilibrium Alfvén velocity at $x=0, z=0$.

Due to the slow $z$-variation of the equilibrium, the gradient along $x$ of the velocity is roughly three times larger at the equatorial plane than at high-latitudes. Thus, we expect the KH instability to develop earlier at low-latitude since its linear growth rate, in a simplified 2D description, is proportional to the equilibrium velocity gradient [27]. In these conditions, the $m_{y}=2$ mode corresponds to the Fast 
Growing Mode at the equator [21], where $m_{y}$ is the wave number along the periodic $y$-direction. The parameters adopted are representative of the "typical" values of the physical quantities that can be observed at the outer magnetosphere. In particular they are related to the dynamics of vortices of typical size much greater than $d_{i}$.

We describe the $\mathrm{KH}$ evolution by adopting a two-fluid, quasi-neutral plasma model. The continuity equation reads $\partial n / \partial t+\nabla \cdot(n \mathbf{U})=0$, where $n$ is the plasma density and $\mathbf{U} \simeq \mathbf{u}_{\mathbf{i}}$ the fluid velocity (neglecting electron inertia). The momentum equation reads

$$
\partial(n \mathbf{U}) / \partial t+\nabla \cdot[(n \mathbf{U} \mathbf{U})+(P \overline{\overline{\mathbf{I}}}-\mathbf{B B})]=0,
$$

with $P=P_{i}+P_{e}+B^{2} / 2$ and we take adiabatic closures given by $\partial\left(n S_{i, e}\right) / \partial t+\nabla \cdot\left(n S_{i, e} \mathbf{u}_{\mathbf{i}, \mathbf{e}}\right)=0$ where $\mathbf{u}_{\mathbf{i}, \mathbf{e}}$ are the ion and electron fluid velocities and $S_{i, e}=P_{i, e} n^{-5 / 3}$. The magnetic field $\mathbf{B}$ is advanced by solving the Faraday equation and the electric field $\mathbf{E}$ is calculated by means of a resistive Ohm's law, $\mathbf{E}=-\mathbf{u}_{\mathbf{e}} \times \mathbf{B}+\eta \mathbf{J}$, with $\eta=10^{-3}$, where we neglect the term $\nabla P_{e} / n$ (for an initial uniform entropy configuration it does not contribute to $\nabla \times \mathbf{E})$. Electron and ion velocities are obtained by combining the fluid velocity $\mathbf{U} \simeq \mathbf{u}_{\mathbf{i}}$ and the current $\mathbf{J} \equiv n\left(\mathbf{u}_{\mathbf{i}}-\mathbf{u}_{\mathbf{e}}\right)=\nabla \times \mathbf{B}$ (we neglect the displacement current). In order to follow the field line connectivity during the plasma evolution we define a passive tracer $\zeta$ that is simply advected by the ion velocity field. At $t=0$ $\zeta$ is constant along field lines, i.e. $\nabla \zeta \cdot \mathbf{B}=0$, has the same spatial scalelength of the velocity field and is equal to $\left.\zeta(x, y, z)\right|_{t=0}=0.6+0.4 \tanh \left[A_{e q, y}(x, z) / L_{u}\right]$. In this way the value 0.2 corresponds to the magnetospheric plasma flowing at $-U_{0} / 2$ and 1.0 to the solar wind plasma flowing at $+U_{0} / 2$.

These equations are integrated numerically with periodic boundary conditions in the $(y, z)$ plane and open transparent conditions in the $x$-direction (see [17] for details). The value of resistivity, that is taken to combine within a fluid description all possible collisional and kinetic phenomena that allow magnetic reconnection at small scales, is normalized using $d_{i}$. For the early evolution of the $\mathrm{KH}$ vortices the scales involved are much greater, meaning that the "renormalized" $\eta$-value should be considered much smaller. Indeed we do not observe any appreciable magnetic diffusion inside the vortices. On the contrary, once current sheets of typical width of $d_{i}$ are generated, the reconnection rate is dominated by the Hall term in the generalized Ohm's law and it is expected to depend smoothly on resistivity [18]. These results have been widely tested in $2 \mathrm{D}$ simulations of magnetized $\mathrm{KH}$ instability [16].

In Fig.1, left frame, we show in red the magnetospheric field lines, in blue the solar wind lines and the passive trace $\zeta$ representing the transition between the magnetospheric blue plasma $(\zeta=0.2)$ and the solar wind red plasma $(\zeta=1.0)$ at $t=410$. We see two rolled-up vortices generated by the $\mathrm{KH}$ instability, lying in the equatorial plane, while no vortices develop at high-latitude.

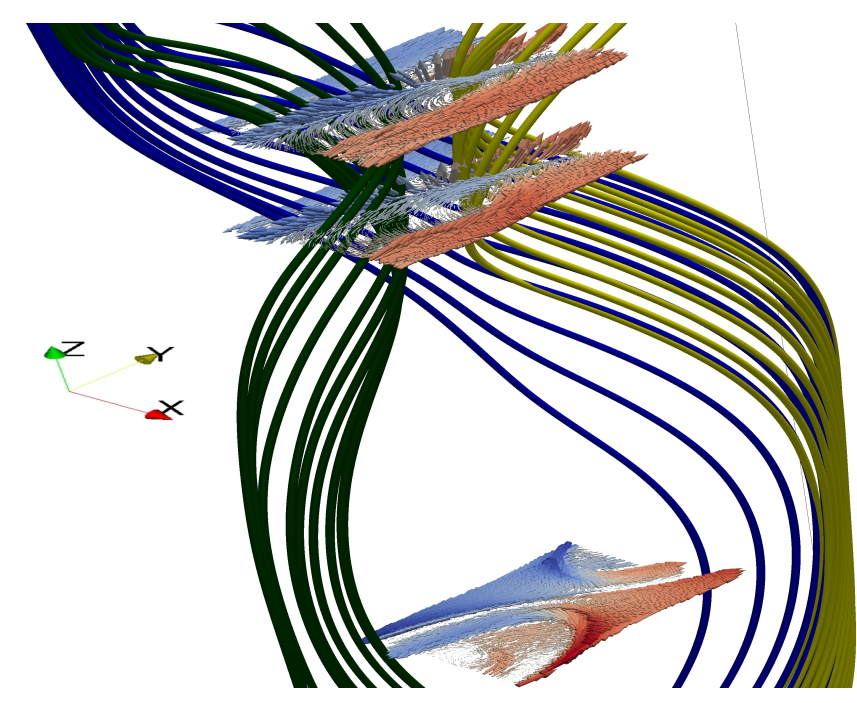

Fig. 2: (color online). Detailed view of the $\mathrm{X}$-point showing magnetic field lines and $\mathbf{u}_{\mathbf{i}}$-vectors in $x, y$-planes at $z=$ $0,75,115$, colored in blue/red if $u_{i, y} \lessgtr 0$, at $t=430$.

Due to the frozen-in condition, the magnetospheric (blue) field lines, starting at $z= \pm L_{z} / 2$, cross the blue arms of the vortices at the equatorial plane and finally reach their symmetric magnetospheric region at $z=\mp L_{z} / 2$, still maintaining their original connectivity since no reconnection process has yet taken place. Solar wind (red) field lines undergo a similar evolution. However, the magnetic field advection bends and arches the field lines, thus creating extended current sheets at mid-latitudes (light green region in Fig.1, left frame) where reconnection can now take place. It is worth noticing that the differential advection between field lines is driven not only by the rolling-up of the vortices but mainly by the motion of the field lines themselves, anchored in the moving plasma at high latitude, with respect to their anchoring in the vortices at rest in the equatorial region. Indeed, in the numerical reference frame, the magnetosphere and solar wind plasmas move in the opposite direction (asymptotically $\pm V_{0}$ ), while the phase velocity of the vortices is zero. Looking at the northern hemisphere we can clearly see the magnetic structure of the current layer at mid-latitude at $x \simeq 0, y \simeq L_{y} / 6, t=410$ where reconnection is occurring. In this picture, blue and red lines are those not yet reconnected and that still maintain their original connectivity to the magnetosphere and to the solar wind, respectively. On the contrary, green lines represent those that have reconnected once in the northern hemisphere and that connect the magnetospheric region at $z=L_{z} / 2$ to the solar wind red arms of the $\mathrm{KH}$ vortices in the equatorial plane at $z=0$ and finally to solar wind regions $z=-L_{z} / 2$. In the same way once-reconnected ochre lines connect the solar wind at $z=L_{z} / 2$ to the magnetosphere at $z=0,-L_{z} / 2$.

We can describe the reconnection region as a nearly $2 \mathrm{D}$ structure with a well defined guide field $B_{z}$, an inverted planar magnetic field given by the blue and 

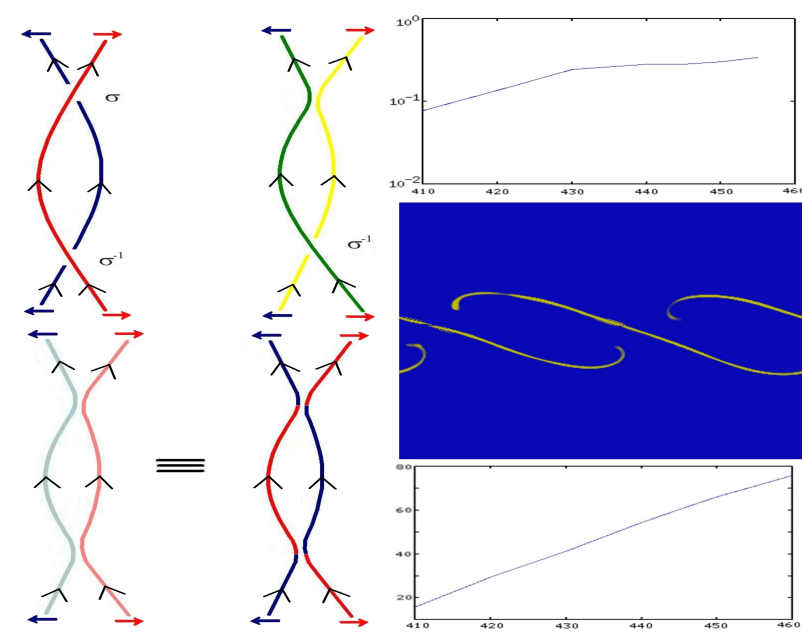

Fig. 3: (color online). Left frame: a schematic representation of the double-reconnection process at mid-latitudes. Right frame: temporal evolution of $B_{\max }=\max (\mathbf{B} \cdot \nabla \zeta /|\nabla \zeta|)$ (top), intersection at $t=430$ (yellow domain) of the regions where the magnetic connectivity has changed with the $z=87$ plane (middle) and temporal evolution of the area $S_{\lambda}$ of this intersection per KH-wavelength.

red $x, y$-components of the magnetic field lines drawn in Fig.1, an $X$-line that bends along $z$ and a current sheet given by $J_{z}$ with a typical length along the $z$ direction $\left(L_{\text {guide field }} \sim 40 d_{i}\right)$ much greater than its width $\left(L_{\text {magnetic shear }} \sim d_{i}\right)$ and its extension along the inverted magnetic field ( $L_{\text {inverted field }} \sim 10 d_{i}$ ). Magnetic reconnection takes place along the entire current sheet, i.e. $L_{X \text {-line }} \sim L_{\text {guide field }}$.

Details of the northern reconnection zone at $t=430$ are shown in Fig.2. We adopt the same color code for magnetic field lines used in Fig.1 but, for the sake of clarity, we do not plot the red lines belonging to the solar wind. The ion velocity field $\mathbf{u}_{\mathbf{i}}$ in the horizontal sections at different heights is represented by blue and red arrows. These arrows show the outflow structures (their $z$-component is related to the ion contribution to the current) that advect the reconnected green and ochre lines away from the $X$-line. At mid-latitude these outflow structures are superimposed to the velocity field generated by the hydrodynamic instability while they are not present in the velocity pattern of the vortices in the equatorial region where the $\mathrm{KH}$ instability is stronger. This indicates that reconnection develops as an independent secondary instability at mid-latitude, taking advantage of the favorable conditions created by the large-scale KH instability, and that it does not proceed as forced reconnection simply driven by the velocity field imposed by the growing KH instability [28].

In Fig.1, right frame, we can see that magnetic reconnection proceeds almost simultaneously in the northern and southern hemispheres creating grey field lines that are connected to the magnetosphere in both hemispheres at high latitude but that cross the solar wind region (red vortex arm) at low latitude. Grey lines are further away, along the outflow, from the northern $X$-line compared to green lines. Since the outflow advects the reconnected lines far away from the reconnection region we can relate the distance from the X-line to the time in the past when lines reconnected. Grey field lines thus originate from green-type lines that reconnected once in the northern hemisphere (at a previous time compared to the green lines plotted in this figure) and that have reconnected again at a subsequent time in the southern hemisphere, still at mid-latitude. The delay between the two different reconnection processes is comparable to the reconnection time, as given by $\tau_{\text {rec }}^{-1} \simeq \gamma_{\text {rec }}=0.06$. This value is obtained from the temporal evolution of $B_{\max }=\max (\mathbf{B} \cdot \nabla \zeta /|\nabla \zeta|)$ (Fig.3, righttop frame) and is compatible with the value expected for fast magnetic reconnection [18]. The quantity $\mathbf{B} \cdot \nabla \zeta /|\nabla \zeta|$, which vanishes outside narrow (nearly $2 \mathrm{D}$ ) regions where reconnection has occurred, gives the component of the magnetic field perpendicular to the magnetospheric frontier. This quantity generalizes the $x$-component of the magnetic field created by the linear evolution of Tearing instability in a $2 \mathrm{D}$ configuration with an equilibrium field given by $B_{0} \tanh (x / l)$, described by eigenfunctions of the form $B_{x, k}(x, y, t)=\tilde{B}_{x, k}(x) e^{i k y} e^{\gamma_{k} t}[7,29-31]$.

This double-reconnection process creates magnetic field lines that are topologically part of the magnetosphere (field lines that close on Earth) but that are connected to the solar wind at low latitude. As a consequence, the associated flux tubes are partially populated by solar wind particles. Double-reconnection at mid-latitude, dynamically allowed by the evolution of the $\mathrm{KH}$ instability, provides a dynamical mechanism for the cold and dense particles of the solar wind to enter the Earth's magnetosphere. This mechanism is not in competition with double lobe reconnection that has been proposed as a mechanism that can close solar wind field lines on Earth $[10,11]$. However invoking the double-reconnection mechanism at mid-latitude has as intrinsic advantage over double lobe reconnection since it is not only favoured by the nearly north-south symmetry of the magnetospheric system but it is actually imposed by the system evolution itself, once reconnection has acted in the northern (southern) hemisphere. Furthermore double mid-latitude reconnection can involve a far greater number of flux tubes (all the tubes flowing around the magnetospheric flanks) compared to the tubes draping over the magnetosphere at its nose position, that are the ones involved in lobe reconnection. A schematic view of double mid-latitude reconnection is given in Fig.3, left frame. The top left graph shows two representative pristine magnetospheric (blue) and solar wind (red) field lines before any reconnection process has taken place. These two different field lines are bent by the differential advection and rolled-up by the equatorial vortices but are not braided. In fact, following the generator formalism [32] a clockwise $\sigma^{-1}$ plus a counterclockwise $\sigma$ elementary line-crossing corresponds 
to the identity operator, i.e. no braiding. As shown in the top right graph, when reconnection occurs in the northern hemisphere the elementary crossing $\sigma$ is cancelled and the once-reconnected green and ochre field lines (colours are changed following the choice made in the left frame) become braided at mid-latitude in the southern hemisphere. Since the southern feet of the green and ochre lines are advected in opposite directions, tailward and solarward respectively, reconnection in the southern hemisphere must take place in order to unbraid the field lines (bottom left graph) and reduce the line stretching. The bottom right graph is equivalent to the bottom left graph where we have simply changed the colors of the double-reconnected grey and pink lines in order to underline the properties of the regions to which they are connected. In particular the blue-red-blue line represents a double-reconnected line whose associated flux tube is connected to Earth in both hemispheres but is partially populated by the solar wind plasma.

Particle signatures of this type have been observed at the dayside magnetopause and have been associated with double lobe reconnection $[11,33-35]$. The same particle signatures were also observed in the near-tail magnetopause within $\mathrm{KH}$ vortices and it was suggested that these particle signatures should be associated to field lines that had undergone lobe reconnection [22].

In order to quantify the impact of double mid-latitude reconnection on plasma transport we consider the time rate at which flux tubes are reconnected and their volume. More specifically we look at regions defined by the condition $\Gamma \geq \Gamma^{*}$, where $\Gamma=|\nabla \zeta \cdot \mathbf{B} /| \mathbf{B} \|$ is taken to represent the spatial rate of change of magnetic connectivity. The threshold value $\Gamma^{*}=0.015$ is determined selfconsistently as the minimal value that satisfies the condition $\Delta \zeta \simeq \Gamma^{*} L^{*} \simeq 0.8$, where $L^{*}$ is the extension of these regions along the field line direction $(\sim \hat{z})$. Consequently all field lines that cross the intersection of these regions with an horizontal plane at mid-latitude (shown in yellow in Fig.3, right-middle frame, for $t=430$ and $z=87$ ) change their connectivity completely from highlatitudes to the equatorial region. The area of the intersection $S_{\lambda}$ in a given KH-wavelength $\lambda$ grows linearly with time (Fig.3, right-bottom frame). In fact field lines, after being reconnected in a region of typical width $d_{i}$, are advected away from the X-line by the plasma outflow and create elongated regions of reconnected lines. The width of these regions is of order $d_{i}$ and does not change significantly in time while their linear extension grows linearly in time in the direction of the outflow with velocity $2 U_{\text {outflow }} \simeq 2 U_{A}$. Thus the volume of plasma exchanged per unit $\mathrm{KH}$ wavelength between open and closed field lines is given by $V_{\lambda}=S_{\lambda} L_{z} / 2$, where $L_{z} / 2$ represents the average distance between the northern and southern reconnection sites, i.e. the length of the double-reconnected lines embedded into the solar wind. If we consider that only one half of $V_{\lambda}$ corresponds to plasma entrance into the magnetosphere and that the magnetospheric density is negligible compared to that of the solar wind, we obtain a mass flux for unit surface given by

$$
\begin{aligned}
J_{\lambda} & \simeq \frac{1}{2} \frac{d_{i} U_{A} L_{z} \rho_{S W}}{\lambda L_{z}} \sim D_{e f f} \frac{\rho_{S W}}{L_{u}} \\
& \Rightarrow D_{\text {eff }} \sim d_{i} U_{A}\left[L_{u} /(2 \lambda)\right]
\end{aligned}
$$

where we have introduced the velocity scalelength $L_{u} \sim$ $3 d_{i}$ of the equilibrium configuration. Taking $U_{A} \simeq$ $300 \mathrm{Km} / \mathrm{s}$ and $d_{i} \simeq 250 \mathrm{Km}$ as typical values of the outer magnetopause we finally obtain $D_{\text {eff }} \sim O\left(10^{9}\right) \mathrm{m}^{2} / \mathrm{s}$, the same order of magnitude of the diffusion coefficient needed to explain the observed mixing in a simplified viscous model [4].

In conclusion in this letter we propose a new dynamical mechanism for creating double-reconnected field lines during the evolution of $\mathrm{KH}$ vortices in the complex $3 \mathrm{D}$ configuration of the flank magnetopause. Finally we note that while this mechanism does not exclude double lobe reconnection, as the two mechanisms can operate at the same time, but provides an intrinsic and efficient mechanism for producing double-reconnected field lines.

$$
* * *
$$

The research leading to these results has received funding from the European Commission's Seventh Framework Programme (FP7/2007-2013) under the grant agreement SWIFF (project $\mathrm{n}^{\mathrm{o}}$ 263340, www.swiff.eu). This work was supported by the Italian Supercomputing Center CINECA under the ISCRA initiative.

\section{REFERENCES}

[1] S. Wedemeyer-Böhm et al., Nature 486, 505 (2012).

[2] H. Hasegawa et al., Nature 430, 755 (2004).

[3] T. Terasawa et al., Geophys. Res. Lett. 24, 935 (1997).

[4] B. U. Ö. Sonnerup, J. Geophys. Res. 85, 2017 (1980).

[5] J. Labelle et al., Space Sci. Rev. 47, 175 (1988).

[6] G.C. Le et al., J. Geophys. Res. 99, 23723 (1994).

[7] B. Coppi, Phys. Lett. 11, 226 (1964).

[8] J.A. Wesson, Nuclear Fusion 30, 2545 (1990).

[9] J.T. Gosling et al., J. Geophys. Res. 96, 14097 (1991).

[10] P. Song et al., J. Geophys. Res. 97, 1411 (1992).

[11] T.G. Onsager et al., J. Geophys. Res. 106, 25467 (2001).

[12] A. Otto et al., J. Geophys. Res. 105, 21175 (2000).

[13] T.K.M. Nakamura et al., Adv. Space. Res. 37, 522 (2006).

[14] K. Nykyri et al., Ann. Geophys 24, 2619 (2006).

[15] M. Faganello et al., Phys. Rev. Lett. 101, 105001 (2008).

[16] M. Faganello, et al., Phys. Rev. Lett. 101, 175003 (2008).

[17] M. Faganello et al., New J. Phys. 11, 063008 (2009).

[18] J. Birn et al., J. Geophys. Res. 106, 3737 (2001).

[19] C. Hashimoto et al., Adv. Space. Res 37, 527 (2006).

[20] K. Takagi et al., J. Geophys. Res. 111 A08202 (2006).

[21] M. Faganello et al., Plasma Phys. Control. Fusion, 54, 124037 (2012).

[22] M.B. Bavassano et al., Ann. Geophys. 28, 893 (2010).

[23] T. Andreussi et al., Phys. Plasmas 19, 052102 (2012). 
[24] Y. Matsumoto et al., Geophys. Res. Lett. 31, L02807 (2004).

[25] M. Faganello et al., Phys. Rev. Lett. 100, 015001 (2008).

[26] A. Tenerani et al., Plasma Phys. Control. Fusion, 53 015003 (2011).

[27] A. Miura, Phys. Plasmas 4, 2871 (1997).

[28] D.A. Knoll et al., Phys. Rev. Lett. 88, 215003 (2002).

[29] H.P. Furth et al., Phys. Fluids 6, 459 (1963).

[30] I. Hofmann, Plasams Phys. 17, 143 (1975).

[31] M. Faganello et al., Phys. Plasmas 17, 062102 (2010).

[32] J.-L Thiffeault, Phys. Rev. Lett. 94, 084502 (2005).

[33] B. Lavraud et al., J. Geophys. Res. 110, A06209 (2005).

[34] M. Fujimoto et al., J. Geophys. Res. 103, 4391 (1998).

[35] J.P. McFadden et al., Geophys. Res. Lett. 35, L17S09 (2008). 